\title{
The Effects of Acute and Chronic Inflammation on the Dynamics of Fluid Shift of Ringer's Solution and Hemodynamics during Surgery
}

\author{
Xiuzhen Lei, ${ }^{a}$ Manli An, ${ }^{a}$ Yulong Wang, ${ }^{a}$ Shenghua Yi, ${ }^{b}$ Yeke Zhu, ${ }^{a}$ Shuangyan $\mathrm{Hu},{ }^{b}$ \\ Yuhong $\mathrm{Li}^{*}, b$ and Shengmei $\mathrm{Zhu}^{*, a}$ \\ ${ }^{a}$ Department of Anesthesiology, the First Affiliated Hospital, College of Medicine, Zhejiang University; Hangzhou \\ 310000, China: and ${ }^{b}$ Department of Anesthesiology and Clinical Research Center, Shaoxing People's Hospital \\ (Shaoxing Hospital of Zhejiang University); Shaoxing 312000, China. \\ Received July 29, 2019; accepted July 5, 2020; advance publication released online August 4, 2020
}

\begin{abstract}
The aim of this study was to investigate the influences of acute and chronic inflammation on the dynamics of fluid shift of Ringer's solution and hemodynamics in patients during surgery. Thirty-seven patients with the American Society of Anesthesiologists (ASA) grades I-II were enrolled and allocated to two study groups according to the type of disease and operation and inflammation, including patients undergoing emergency appendectomy (Acute group, $n=19$ ) and patients undergoing elective cholecystectomy (Chronic group, $n=18$ ). All of the patients were administered $15 \mathrm{~mL} / \mathrm{kg}$ of Ringer's lactated (LR) solution at a constant rate over $35 \mathrm{~min}$ before the induction of anesthesia. Plasma dilution (PD), volume expansion (VE), volume expansion efficiency (VEE), and extravascular volume (EVV) were calculated based on the concentration of hemoglobin within $2 \mathrm{~h}$ post-infusion. Heart rate (HR), arterial blood pressure and urine output were also recorded. PD and VE peaked at the end of infusion, while VEE peaked at the beginning of infusion in all of the patients. After infusion, PD, VE and VEE in the Acute group were all higher than those in the Chronic group $(p<0.05)$. PD and VE were higher during anesthesia or surgery than during awake or non-surgery $(p<0.001)$. The mean arterial pressure (MAP) and diastolic pressure (DBP) in the Acute group were significantly lower $(p<0.001)$ and HR was significantly higher $(p<0.001)$ than in the Chronic group during the study periods. It was suggested that patients with acute inflammation be treated with individualized fluid therapy during surgery.
\end{abstract}

Key words lactated Ringer's solution; acute inflammation; chronic inflammation; anesthesia; volume expansion; hemodynamics

\section{INTRODUCTION}

Seventy percent of patients have absolute blood volume deficiency before surgery due to fasting, bowel preparation and accompanying diseases. ${ }^{1-3)}$ During the induction of anesthesia, due to the vasodilatation effect of general or local anesthetics, there exists a relative blood volume deficiency. $\left.{ }^{4}\right)$ Therefore, all surgical patients require fluid therapy before anesthesia induction. A variety of methods have been used to detect the volume expansion (VE) effect of fluid therapy. In previous studies, ${ }^{3,4)}$ we used hemoglobin $(\mathrm{Hb})$ dilution to reflect the VE of the infused fluid. Ringer's lactated (LR) solution is preoperative main infusion fluid, and a period of time after infusion, in accordance with the principle of $4: 1$ allocation of crystalloid, only $20 \%$ of fluid remains in the intra-vascular space, resulting in VE.

Our previous studies ${ }^{3,4)}$ indicated that the VE of infused crystalloid was more than $1: 1$ on some certain occasions, such as during anesthesia, at early times of infusion and with low blood volume. Multi-factor regression found that hypotension was an independent factor for increased VE. One of our studies $^{3)}$ showed that, compared with epidural anesthesia, the VE of infusion crystalloid was higher during the induction of general anesthesia. Another clinical study ${ }^{4}$ showed that the VE of infused crystalloid was stronger in adults than in children. Additionally, the hemodynamic changes of patients in the two age groups showed different trends post-infusion.

In addition to the aforementioned factors (anesthesia and age), co-administered drugs might also change the VE, such as phenylephedrine and esmolol reducing the VE of LR. ${ }^{5,6}$ Our recent animal study ${ }^{7}$ found that the effect of VE of LR decreased in the early stage of shock. Additionally, phenylephedrine, esmolol and other vasoactive drugs had different effects on VE in animals with different diseases. ${ }^{7-10)}$

Patients with acute appendicitis often have associated systemic inflammatory response syndrome (SIRS), which can be associated with increased capillary permeability before surgery. The reason for VE after crystalloid infusion in SIRS patients remains undetermined. Based on previous studies, it is hypothesized that most of the fluid infused during surgery in SIRS patients accumulates interstitial the space, resulting in lower blood VE. To verify this assumption, we selected patients undergoing emergency laparoscopic appendectomy or elective laparoscopic cholecystectomy to explore the effect of SIRS on VE and hemodynamics after LR infusion.

\section{MATERIALS AND METHODS}

Ethics Statement This study program was discussed and approved by the Ethics Committee of Shaoxing People's Hospital (No. 2015013; official in charge: Yu Qian), and the study was registered at the Chinese Clinical Trial Registry (website: http://www.clictr.org.cn, No. CHICTR-15006063). All patients or guardians were fully informed of the protocol and signed a consent form before participating in the trial.

Subject Selection From March 2015 to April 2017, forty 
patients aged 18-60 years old with body mass index (BMI) 18-25 and grades I-II of the American Society of Anesthesiologists (ASA) were included for elective laparoscopic cholecystectomy (Chronic group, $n=20$ ) and emergency laparoscopic appendectomy (Acute group, $n=20$ ) under general anesthesia. Patients with cardiopulmonary, hepatorenal and endocrine diseases, nervous system diseases, or severe anemia $(\mathrm{Hb}<65 \mathrm{~g} / \mathrm{L})$ were excluded. Patients with intraoperative blood loss of more than $200 \mathrm{~mL}$ or patients received vasopressor or patients who needed to change surgical procedures were also excluded. Additionally, patients with preoperative cognitive impairment, obese patients $($ BMI $>25)$, and pregnant and lactating women were excluded. Both patients taking diuretics and patients taking antihypertensive substances with diuretic properties were also excluded.

Experiment Design This was a single-center, prospective cohort study. The diagnosis of SIRS was based on criteria established by the joint association of the American College of Chest Physicians (ACCP) and Society of Critical Care Medicine (SCCM) in 1991 by meeting at least two of the following indicators: (1) temperature is greater than $38.0^{\circ} \mathrm{C}$ or less than $36.0^{\circ} \mathrm{C}$; (2) heart rate is greater than 90 beat per minute (BPM); (3) respiration rate is greater than $20 \mathrm{BPM}$ or $\mathrm{PaCO}_{2}$ is less than $32 \mathrm{mmHg}(4.3 \mathrm{kPa})$; and (4) leukocyte count is greater than $12.0 \times 109 / \mathrm{L}$ or neutrophil granulocytes (immature cells) are greater than 0.10 . Patients undergoing acute appendectomy who met the SIRS diagnostic criteria and had higher $\mathrm{C}$ reactive protein (CRP) than normal $(10 \mathrm{mg} / \mathrm{mL})$ were included in the Acute group. Patients with elective cholecystitis or cholelithiasis who did not meet these criteria were included in the Chronic group.
Procedure This study was completed in the operating room of Shaoxing People's Hospital. All of the patients fasted for $8 \mathrm{~h}$ before surgery without preoperative medication. After entering the operating theater, all of the patients were given oxygen at $2-4 \mathrm{~L} / \mathrm{h}$. The catheter was placed in the left radial artery for monitoring blood pressure and collecting blood samples for measurements of $\mathrm{Hb}$ and hematocrit (Hct). Anesthesia was induced with propofol $1.5 \mathrm{mg} / \mathrm{kg}$ (Astrazeneca Co., Ltd., U.K.), midazolam $50 \mu \mathrm{g} / \mathrm{kg}$ (Enhua Pharmaceutical Co., Ltd., Jangsu, China), cisatracurium $0.15 \mathrm{mg} / \mathrm{kg}$ (Hengrui Pharmaceutical Co., Ltd., Jiangsu, China), and sufentanil $5 \mu \mathrm{g} / \mathrm{kg}$. After tracheal intubation, the parameters of mechanical ventilation were set as follows: tidal volume (VT) $8 \mathrm{~mL} / \mathrm{kg}$; positive end expiratory pressure (PEEP) $3 \mathrm{~cm} \mathrm{H}_{2} \mathrm{O}$; breathing frequency 12 times/min; I:E ratio $1: 2$; and maintaining end-tidal carbon dioxide partial pressure $\left(\mathrm{P}_{\mathrm{ET}} \mathrm{CO}_{2}\right)$ at $36-44 \mathrm{mmHg}$. Anesthesia was maintained with 1-2\% sevoflurane (Hengrui Pharmaceutical Co., Ltd.) and propofol $6 \mathrm{mg} / \mathrm{kg} / \mathrm{h}$. Additional sufentanil $0.2-0.4 \mu \mathrm{g} / \mathrm{kg}$ and cisatracurium $0.05 \mathrm{mg} / \mathrm{kg}$ were administered as required. The depth of anesthesia was monitored using a bispectral index (BIS) sensor applied to the forehead. The signal was recorded on a BIS monitor Model A-2000TM (Aspect Medical Systems, Natick, MA, U.S.A.), and the level of anesthesia was guided to reach a BIS value between 40 and 60 . After the induction of anesthesia, an indwelling urinary catheter was placed, the bladder emptied, and a urinary bag connected. Then, Ringer's lactate was administered at a volume of $15 \mathrm{~mL} / \mathrm{kg}$ over $35 \mathrm{~min}$ via a constant-rate infusion pump (ICE 601-1; Abbott Laboratories, Chicago, IL, U.S.A.). After infusion, there was no fluid infusion except for intravenous administration of drugs and diluted heparin fluid to flush the arterial

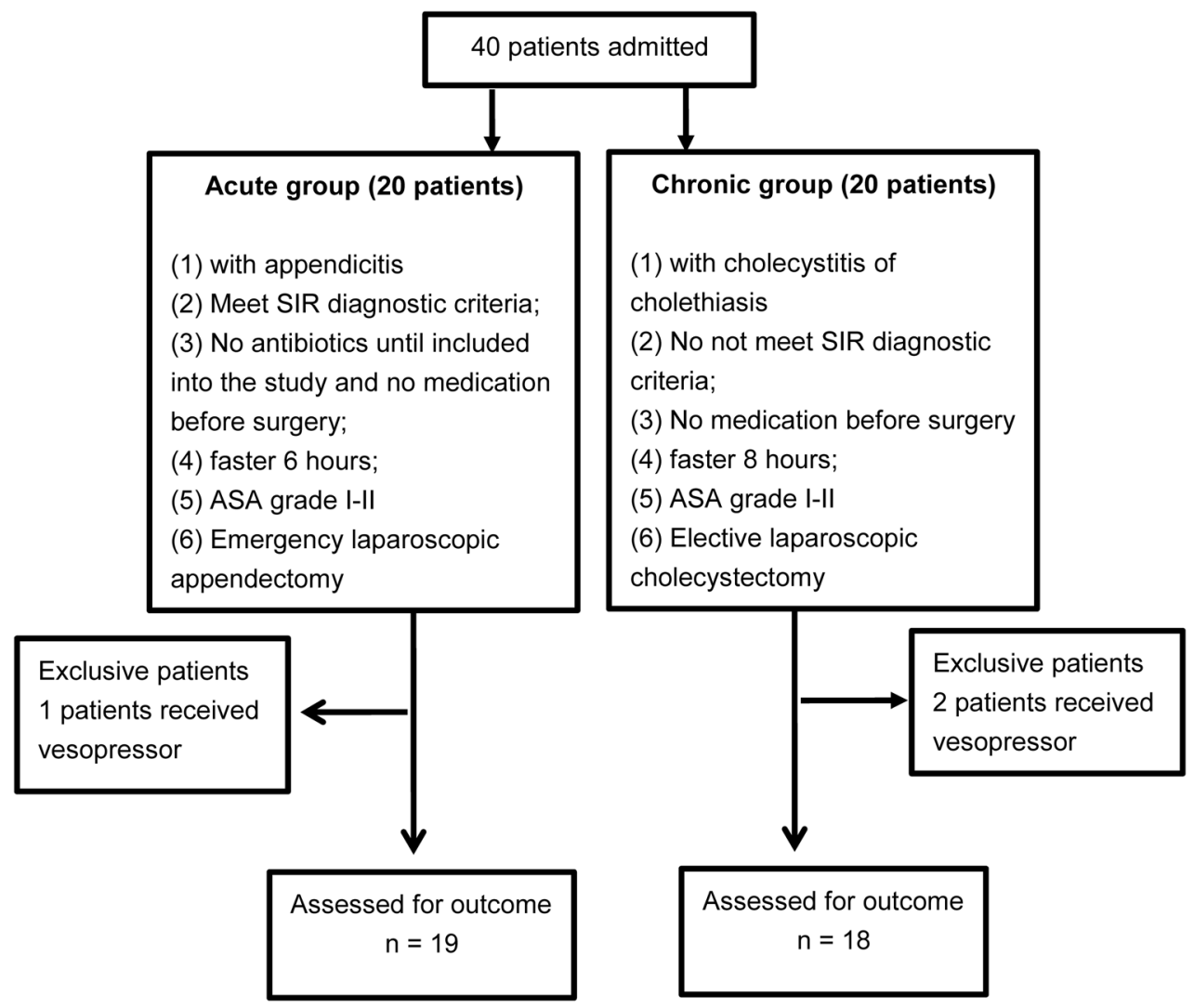

Fig. 1. Flow Diagram 
tube. During the experiment, ephedrine 5-10 mg was given i.v. when mean arterial pressure (MAP) fell to less than $65 \mathrm{mmHg}$ and atropine 0.5 i.v. when the heart rate was less than $50 \mathrm{bpm}$. Patients were transferred to postoperative anesthesia care unit (PACU) after surgery (Fig. 1).

Indicators and Measurements Electrocardiogram (ECG), heart rate $(\mathrm{HR})$, pulse oxygen saturation $\left(\mathrm{SpO}_{2}\right)$, systolic blood pressure (SBP), diastolic blood pressure (DBP), MAP, and $\mathrm{P}_{\mathrm{ET}} \mathrm{CO}_{2}$ were continuously monitored using a multi-function monitor (Dates-ohmeda, the Netherlands). Anesthesia depth was controlled by BIS values. Arterial blood samples $(1 \mathrm{~mL})$ were collected in heparinized 2-mL syringes every 5 min during the 1 st $60 \mathrm{~min}$ post-infusion and every $10 \mathrm{~min}$ from 61 to $120 \mathrm{~min}$ for measurements of $\mathrm{Hb}$ and Hct using a Gas Analyzer (GE Premier 3000, Lexington Equipment Laboratory, IL, U.S.A.). The first sample for each patient was measured twice to calculate the mean value to detect the variation coefficient of $1.5 \%$. Patients' urine volumes (UV) and intraoperative blood loss were recorded at the end of the experiment.

Calculations Baseline plasma volume $\left(\mathrm{PV}_{0}\right)$ was estimated from the body weight and height of each patient based on a previous study ${ }^{11)}$ (Formula 1). Plasma dilution (PD) was calculated using the values of $\mathrm{Hb}$ and Hct at both baseline $\left(\mathrm{Hb}_{0}\right)$ and the specific time $\left(\mathrm{Hb}_{\mathrm{t}}\right)$ after infusion (Formula 2). Plasma volume at a specific time $\left(\mathrm{PV}_{\mathrm{t}}\right)$ was calculated by $\mathrm{PV}_{0}$, $\mathrm{Hb}_{0}$ and $\mathrm{Hb}_{\mathrm{t}}$ (Formula 3). VE, in response to fluid infusion, was equal to the difference between $\mathrm{PV}_{\mathrm{t}}$ and $\mathrm{PV}_{0}$ (Formula 4), while the infusion volume retention in the interstitial space, extravascular volume (EVV), was equal to the infusion volume (IV) minus the VE and UV (Formula 5). We also calculated the volume expansion efficiency (VEE) using VE divided by IV (Formula 6) and the urine output efficiency using UV divided by IV. All of the volumes were expressed as $\mathrm{mL} / \mathrm{kg}$, and the rate of urine output was expressed as the urine per $\min (\mathrm{mL} / \mathrm{min})$.

$$
\begin{aligned}
& \text { Formula 1: } \mathrm{PV}_{0}(\mathrm{~mL}) \\
& \quad=0.03308 \times \text { weight }(\mathrm{kg})+0.35611 \times \text { height }^{3} \mathrm{~m}+0.1833 \\
& \text { Formula 2: } \mathrm{PD}=\left(\frac{[\mathrm{Hb}]_{0}}{[\mathrm{Hb}]_{\mathrm{t}}}-1\right) /\left(1-[\mathrm{Hb}]_{\mathrm{t}}\right) \\
& \text { Formala 3: } \mathrm{PV}_{\mathrm{t}}=\mathrm{PV}_{0} \times \frac{[\mathrm{Hb}]_{0}}{[\mathrm{Hb}]_{\mathrm{t}}}
\end{aligned}
$$$$
\text { Formula 4: } \mathrm{VE}(\mathrm{mL})=\mathrm{PV}_{\mathrm{t}}-\mathrm{PV}_{0}
$$$$
\text { Formula 5: } \mathrm{EVV}=\mathrm{IV}-\mathrm{VE}-\mathrm{UV}
$$

Formula 6: VEE $=\mathrm{VE} / \mathrm{IV}$
Statistical Analysis Statistical analysis was performed using SPSS software, version 18 (SPSS, Inc., Chicago, IL, U.S.A.), and GraphPad Prism software, version 7 (GraphPad Software, U.S.A.). Numerical variables are expressed as the mean \pm standard deviation (S.D.) or the median (interquartile range) when appropriate. Categorical data (incidence data) were presented as the number or percentage. Means when normally distributed were analyzed by Student's $t$-test. Medians with skewness data were analyzed by the Mann-Whitney $U$ test. Incidence data were analyzed by the Chi-square test or Fisher's exact test when appropriate. $p<0.05$ was considered statistically significant. Sample size estimation was based on an S.D. of $1 / 3$ of the mean, according to the trial study. Power was given at 0.85 to detect a $35 \%$ difference at $p<0.05$ with use of $\mathrm{G} *$ Power software, version 3.0.10. It was then estimated that a minimum of 19 subjects would be necessary

\section{RESULTS}

Demographic Data, Fluid Input and Output Totally three patients (one in the Chronic group and two in the Acute group) who received vasopressor agent (ephedrine, $6 \mathrm{mg}$ i.v.) during study were excluded. A total of 37 patients were included in the analysis, including 18 who underwent laparoscopic cholecystectomy for cholecystitis or cholelithiasis in Chronic group and 19 who underwent laparoscopic appendectomy for acute appendicitis in Acute group. All of the patients with acute appendicitis surgery were operated on within $12 \mathrm{~h}$ of the onset of the disease with average $6 \mathrm{~h}$ or so. No severe complications occurred in any of the patients during the experiment. There was no significant difference in the patients' demographic data, infusion volume or urine volume between the two groups $(p>0.05)$ (Table 1$)$. The preoperative serum albumin concentration of the two groups was $43.4 \pm 4.1 \mathrm{~g} / \mathrm{L}$ in the chronic group and $41.9 \pm 4.9 \mathrm{~g} / \mathrm{L}$ in the acute group, respectively. There was no significant difference between the two groups $(p=0.300)$.

Hemodilution, Vascular and Extravascular VE After fluid infusion, $\mathrm{Hb}$ started to decrease with the highest point of $2.4 \mathrm{~g} / \mathrm{L}$. Repeated measures ANOVA showed that the PD of the patients in two groups reached the peak dilution at the time of fluid infusion ending, and then it decreased (Fig. 2). PD was calculated based on $\mathrm{Hb}$ and Hct at 5, 10, 35, 60, 90 , and $120 \mathrm{~min}$ after fluid infusion. There was no statistically significant difference in PD between the two groups at the above time points $(p>0.05)$. During the time of fluid infusion (0-35 min), the PD in the two groups was similar $(p=0.261)$, while after infusion $(40-120 \mathrm{~min})$, the $\mathrm{PD}$ in the Acute group was higher than in the chronic $(p=0.037)$

\begin{tabular}{|c|c|c|c|c|c|c|c|c|c|c|}
\hline Group & $\begin{array}{l}\operatorname{Age}^{a)} \\
(\mathrm{yrs})\end{array}$ & $\begin{array}{l}\operatorname{Sex}^{b)} \\
(\mathrm{M} / \mathrm{F})\end{array}$ & $\begin{array}{l}\text { Weight }^{a)} \\
\quad(\mathrm{kg})\end{array}$ & $\begin{array}{l}\left.\text { Height }^{a}\right) \\
\quad(\mathrm{cm})\end{array}$ & $\mathrm{BMI}^{a)}$ & $\begin{array}{c}\text { Surgical } \\
\text { time }^{a)}(\mathrm{min})\end{array}$ & $\begin{array}{l}\text { Urine output }{ }^{c)} \\
\quad(\mathrm{mL} / \mathrm{kg})\end{array}$ & $\begin{array}{c}\text { Urine rate } \\
(\%)\end{array}$ & $\begin{array}{c}\left.\text { Blood loss }^{c}\right) \\
(\mathrm{mL})\end{array}$ & $\begin{array}{c}\text { Infusion } \\
\text { volume }^{a)}(\mathrm{mL})\end{array}$ \\
\hline Chronic group & $46 \pm 12$ & $8 / 10$ & $57 \pm 12$ & $162 \pm 9$ & $22 \pm 4$ & $52 \pm 23$ & $1.5(0.9,2.3)$ & $0.03(0.02,0.06)$ & $20(19,25)$ & $860 \pm 172$ \\
\hline Acute group & $46 \pm 16$ & $8 / 11$ & $58 \pm 9$ & $164 \pm 8$ & $22 \pm 3$ & $44 \pm 17$ & $1.4(0.9,1.7)$ & $0.03(0.02,0.08)$ & $20(20,20)$ & $866 \pm 127$ \\
\hline$p$-Values & 0.868 & 0.999 & 0.863 & 0.712 & 0.702 & 0.177 & 0.938 & 0.417 & 0.727 & 0.863 \\
\hline
\end{tabular}
(Table 2). The two groups had the same VE/kg and VEE at each time point $(p>0.05)$. VE gradually increased in both

Table 1. Comparison of Patients' Data and the Amounts of Input and Output

a) Data presented as the mean \pm standard deviation (S.D.). b) Data presented as counts $c$ ) Data presented as median (25th-75th). 

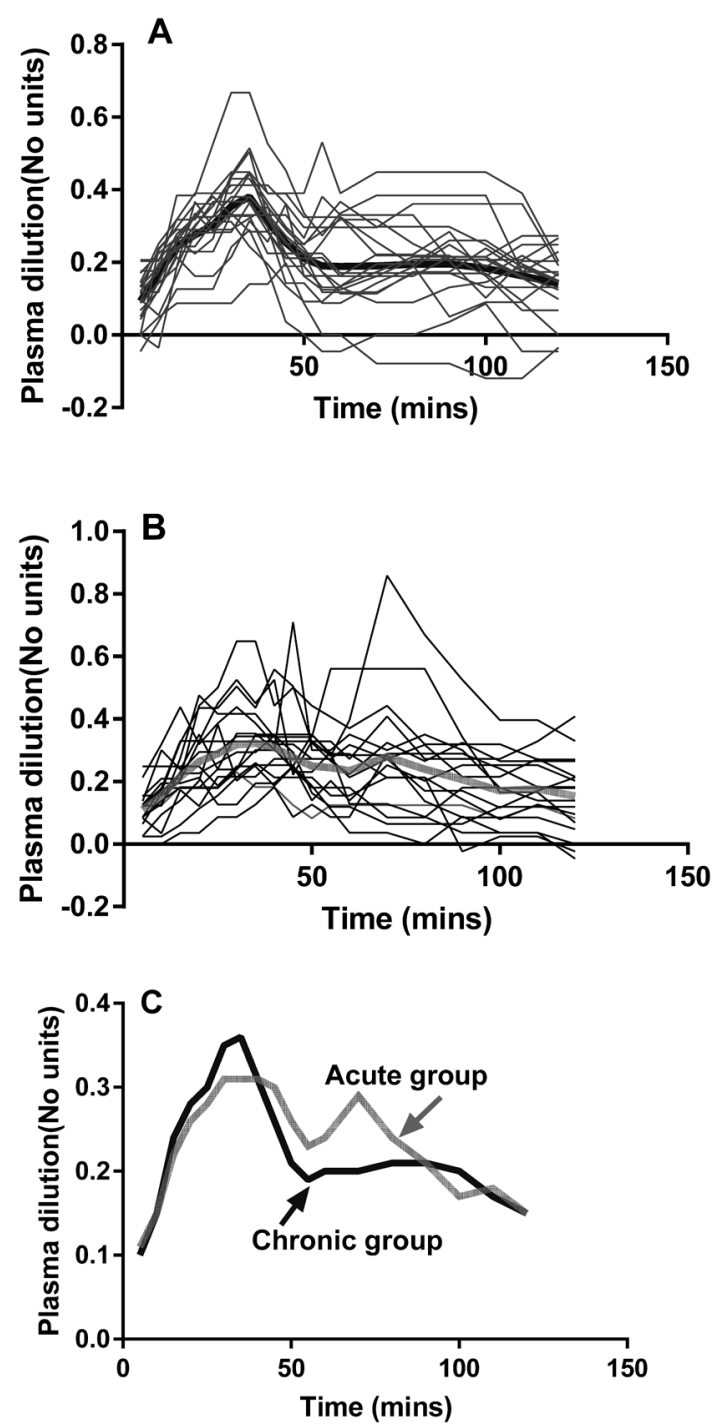

Fig. 2. Plasma Dilution (Thin Lines) during and after 35-min Infusion of $15 \mathrm{~mL} / \mathrm{kg}$ Ringer's Lactate Solution in Individual Patients in the Chronic (A) and Acute (B) Groups

The thick lines are the median values of patients in the Chronic group (black, A and C) or the Acute group (gray, B and C). Superposition of median plasma dilution in the chronic and Acute groups $(\mathrm{C})$.

groups, reaching the peak value at the end of fluid infusion (35 min after infusion) and then decreasing. Five mins after infusion, VEE reached the peak level (Chronic group 1.03; Acute group 1.30) and decreased significantly at each time point $(p<0.01)$. The VEE value was even greater than $100 \%$ 5 min after infusion and decreased down to about $50 \%$ at $35 \mathrm{~min}$ after infusion (Chronic group 0.53; Acute group 0.61). So both groups had higher VEE during infusion (0-35 min), compared with after infusion $(40-120 \mathrm{~min})(p<0.01)$. In addition, there was no difference in VE or VEE between the two groups $(p>0.05)$ during both time period of infusion and the whole experiment, while PD $(p=0.037) \operatorname{VE}(p=0.013)$ and $\operatorname{VEE}(p=0.012)$ after infusion in the Acute group were higher than in the chronic. Compared with during infusion, Both PD and VE in Chronic group decreased significantly after infusion $(p<0.01)$, while these two parameter were similar (Table 2). EVV in the Chronic and Acute group was, respectively, $9.6 \mathrm{~mL} / \mathrm{kg}$ vs. $8.9 \mathrm{~mL} / \mathrm{kg}(p=0.506)$.

Effects of Anesthesia on Hemodilution and Vascular and
Extravascular VE All of the data of the 40 patients were divided into two groups according to the state of being awake or under anesthesia, and then PD, VE and VEE were compared. It was shown that PD $(0.26 v s .0 .20, p<0.001)$ and VE $(6.3$ vs. $5.0 \mathrm{~mL} / \mathrm{kg}, p<0.001)$ during anesthesia were significantly higher than in the awake group (Figs. 3A, B), while VEE in the two states was similar $(0.53$ vs. $0.53, p=0.878)$ (Fig. $3 \mathrm{C})$. In addition, the PD and VE were significantly higher in both the Chronic and Acute group during anesthesia compared to when awake $(p<0.01)$, while VEE was not affected $(p>0.05)$. Further comparison showed that three parameters in both state of anesthesia an awake were comparable in the two groups $(p>0.05)$, as shown in Table 2 .

Effects of Surgery on Hemodilution and Vascular and Extravascular VE All of the data of the 40 patients were divided into two groups according to the state of being surgery or non-surgery. It was shown that PD (0.26 vs. 0.20, $p<0.001)$ and $\operatorname{VE}(6.2$ vs. $5.0 \mathrm{~mL} / \mathrm{kg}, p<0.001)$ during surgery were significantly higher than non-surgery (Figs. 3A, $\mathrm{B})$, while VEE in the two states was comparable (0.52 vs. $0.54, p=0.702$ ) (Fig. 3C). In addition, the PD and VE were significantly higher in both the Chronic and Acute group during anesthesia compared to when awake $(p<0.01)$, while VEE was not affected $(p>0.05)$. Further comparison showed that three parameters during both surgery and non-surgery were comparable in the two groups ( $p>0.05)$, as shown in Table 2.

Hemodynamics The baseline MAP and SBP were comparable between the Chronic and Acute group $(p>0.05)$, but DBP in the Chronic group was higher than in the Acute group (75 vs. 68, $p=0.031$ ). During the whole experiment $(0 \mathrm{~min}-120 \mathrm{~min})$, after infusion and when the patients were awake, except SBP $(p>0.05)$, MAP and DBP in the Chronic group were higher than in the Acute group $(p<0.05)$. When patients were under anesthesia, MAP, DPB and SBP were comparable in both Chronic and Acute group $(p>0.05)$. There was no effect of anesthesia on the blood pressure in patients from both Chronic and Acute group $(p>0.05)$, as shown in Table 3 and Fig. 4A. The baseline HR in the Acute group was higher than in the Chronic group (92 vs. 82, $p=0.044)$, and this trend was maintained during the whole experiment and at each time point. Additionally, BP in all of the patients did not change after infusion $(p>0.05)$, while HR decreased significantly $(p<0.001)$. HR decreased significantly in all patients when they were under anesthesia compared with awake $(p<0.001)$. (Table 3 and Fig. 4B)

\section{DISCUSSION}

After fluid infusion, there are three main outcomes. First, the fluid stays in the blood vessels to result in VE. Second, the fluid distributes to the interstitial space, from which it returns to the blood vessels at a certain rate. If the fluid returns to the blood vessels more slowly than it goes to the interstitial space, it can accumulate in the interstitial space and cause tissue edema. Third, the fluid is excreted outside the body through the kidneys. In the present study, it was found that the maximum plasma dilution (PD) appeared at the end of infusion ( 35 min after infusion), and then VE also reached its peak, while volume expansion efficiency (VEE) presented a different trend, reaching a peak at the beginning of infusion and then decreasing, consistent with our previous studies. ${ }^{3,12,13)}$ We also 
Table 2. Comparison of Patients' Plasma Dilution, VE, and VEE

\begin{tabular}{|c|c|c|c|}
\hline & Chronic group & Acute group & $p$-Values \\
\hline \multicolumn{4}{|l|}{ Plasma dilution, PD (no unit) } \\
\hline During infusion (0-35 min) & $0.26 \pm 0.14$ & $0.24 \pm 0.13$ & 0.261 \\
\hline After infusion $(40-120 \mathrm{~min})$ & $0.21 \pm 0.12^{\# \#}$ & $0.23 \pm 0.14$ & 0.037 \\
\hline During experiment $(0-120 \mathrm{~min})$ & $0.23 \pm 0.13$ & $0.24 \pm 0.14$ & 0.360 \\
\hline Awake & $0.20 \pm 0.11$ & $0.21 \pm 0.14$ & 0.630 \\
\hline Anesthesia & $0.25 \pm 0.14^{* *}$ & $0.26 \pm 0.13^{* *}$ & 0.457 \\
\hline During non-surgery & $0.20 \pm 0.12$ & $0.21+0.14$ & 0.548 \\
\hline During surgery & $0.26 \pm 0.13^{* *}$ & $0.26 \pm 0.14 * *$ & 0.398 \\
\hline \multicolumn{4}{|l|}{ Volume expansion, VE (mL/kg) } \\
\hline During infusion $(0-35 \mathrm{~min})$ & $6.1 \pm 3.2$ & $5.8 \pm 3.2$ & 0.411 \\
\hline After infusion (40-120 min) & $5.0 \pm 3.1^{\# \#}$ & $5.8 \pm 3.3$ & 0.013 \\
\hline During experiment $(5-120 \mathrm{~min})$ & $5.4 \pm 3.2$ & $5.8 \pm 3.3$ & 0.157 \\
\hline Awake & $4.8 \pm 2.7$ & $5.1 \pm 3.2$ & 0.422 \\
\hline Anesthesia & $6.0 \pm 3.4^{*}$ & $6.5 \pm 3.2 *$ & 0.213 \\
\hline During non-surgery & $5.0 \pm 2.9$ & $5.1 \pm 3.2$ & 0.393 \\
\hline During surgery & $6.2 \pm 3.2 * *$ & $6.5 \pm 3.2 * *$ & 0.186 \\
\hline \multicolumn{4}{|l|}{ Volume expansion efficiency, VEE } \\
\hline During infusion $(0-35 \mathrm{~min})$ & $0.79 \pm 0.44$ & $0.79 \pm 0.47$ & 0.952 \\
\hline After infusion (40-120 min) & $0.32 \pm 0.20^{\# \#}$ & $0.39 \pm 0.22^{\# \#}$ & 0.012 \\
\hline During experiment $(0-120 \mathrm{~min})$ & $0.51 \pm 0.39$ & $0.54 \pm 0.39$ & 0.259 \\
\hline Awake & $0.53 \pm 0.44$ & $0.53 \pm 0.46$ & 0.967 \\
\hline Anesthesia & $0.50 \pm 0.33$ & $0.56 \pm 0.32$ & 0.106 \\
\hline During non-surgery & $0.54 \pm 0.45$ & $0.53 \pm 0.46$ & 0.973 \\
\hline During surgery & $0.52 \pm 0.39$ & $0.56 \pm 0.39$ & 0.087 \\
\hline
\end{tabular}

Data are expressed as the mean \pm S.D.; ${ }^{*} p<0.05$, ${ }^{*} p<0.01$ vs. awake or during non-surgery; ${ }^{\#} p<0.01$ vs. during infusion (0-35 min).

found that there was no difference in PD, VE or VEE between the patients with chronic and acute inflammation during infusion, but after infusion, three volume effect indices in the Acute group were all higher than in the Chronic group (Table 2). There was no difference between the two groups in extravascular fluid or fluid clearance. There is the second peak in the Acute group about $70 \mathrm{~min}$ post-infusion when the surgery finished for most of patients, but they were still under anesthesia. The residual effect of anesthetics might cause vasodilation or myocardial depression. At the same time, the acute group had lower blood pressure and a faster heart rate, suggesting a possible hypovolemia accompanied by higher values of VE and VEE in Acute group than in the Chronic group during this period.

We used a two-compartment volume model to simulate the relationship of plasma dilution and time (D-T curve). It showed that the fluid distribution rate constant $\left(k_{12}\right)$ from the central fluid space (intravascular) to the peripheral fluid space (extravascular) was nearly identical between patients with cholelithiasis (Chronic group) and patients with appendicitis (Acute group), but the rate constant of the opposite $\left(k_{21}\right)$ was slower in the Acute group, indicating that fluid accumulation in the interstitial space might occur after surgery in acute appendicitis. ${ }^{14)}$ Drobin and $\mathrm{Hahn}^{15)}$ found that, after infusion of crystal fluid in pre-eclampsia patients, the VE decreased, and the extravascular fluid accumulation and clearance of fluid increased. Our previous animal experiments showed that, in septic sheep with moderate hypovolemia and hypotension, the fluid clearance through the kidneys slowed and trended to distribute to the interstitial space, resulting in tissue edema.' Another previous study demonstrated that fluids were administered during anesthesia induction ${ }^{16)}$ or when awake ${ }^{3)}$ in patients undergoing major surgery, the VEE was more than $100 \%$ at $5 \mathrm{~min}$ after infusion. In the present study, $5 \mathrm{~min}$ postinfusion, all of the patients were awake; VEE was also greater than $100 \%$. This phenomenon was called "interstitial fluid autologous blood transfusion." As early as 1969, Lanne et al. ${ }^{17)}$ reported that such a phenomenon can occur when the hydrostatic pressure of capillaries decreases. In this study, even at 120 min post-infusion, the VEE was still more than $20 \%$, indicating that the distribution balance of crystalloid $(1: 4$ distribution rule) was not reached. In another our previous study, ${ }^{18)}$ VEE was less than $100 \%$ after LR infusion and before anesthesia induction for minor surgery in both adults and children. VEE reached a distribution balance of $1: 4$ at $30 \mathrm{~min}$ post-infusion in children and at $90 \mathrm{~min}$ post-infusion in adults. Thus far, the related factors affecting the expansion efficiency of crystalloid are not clear, and further study is needed.

Our previous studies have found that PD, VE and VEE triggered by crystalloid infusion during the induction of general anesthesia were significantly higher than those during epidural anesthesia. ${ }^{16)}$ In the present study, the volume effects of LR were compared between anesthesia and being awake in each patient. The results showed that PD and VE in the anesthesia group were higher than in the awake group, but VEE was similar between the two states. Under anesthesia or during awake, three parameters were comparable between the Chronic and Acute group, which showed that inflammatory state, likely including surgery procedure or other disease characteristics have no effect on the Ringer's VE. Volume kinetics analysis demonstrated that the reduced fluid clearance of infused LR under anesthesia was $1 / 10$ of that under consciousness, indicating that the infused fluid was reduced through renal clearance and might be remained in the blood vessels to play a role 


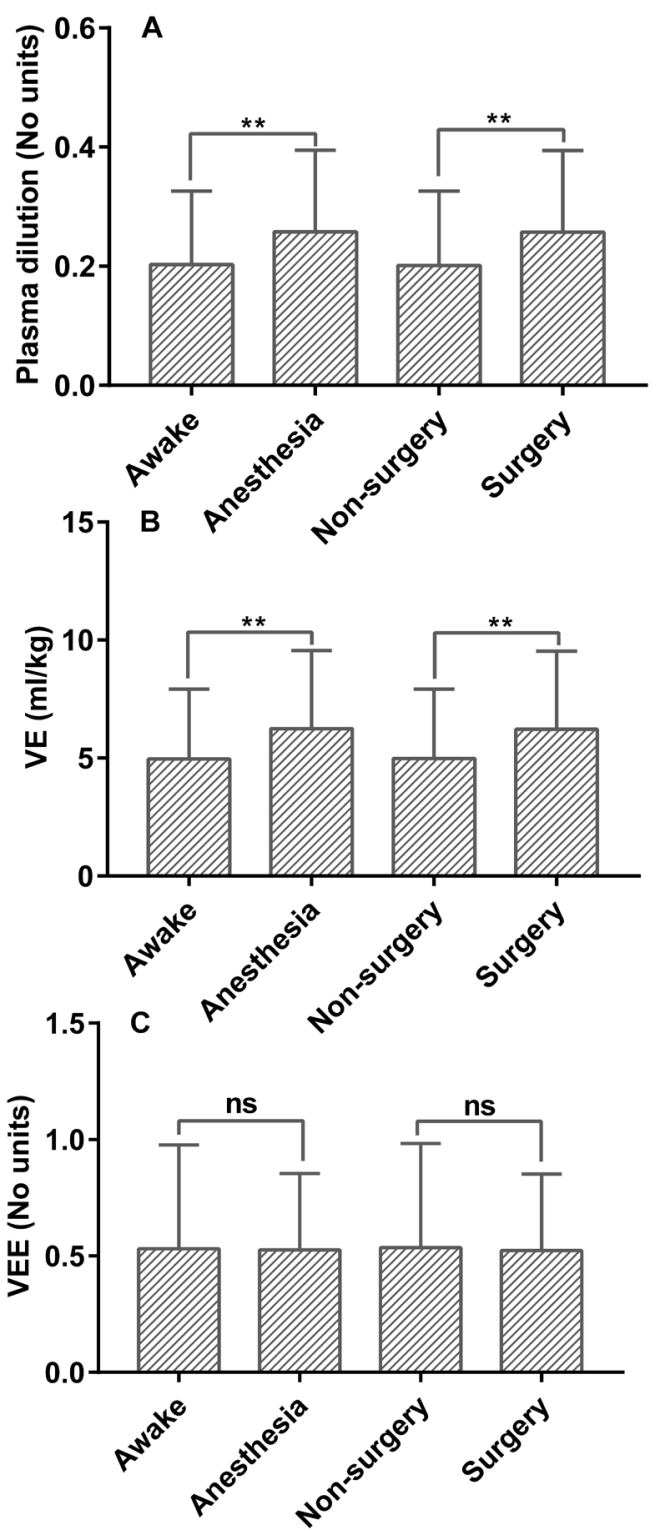

Fig. 3. Comparison of Plasma Dilution (A), Volume Expansion (VE, B) and Volume Expansion Efficiency $(\mathrm{VEE}, \mathrm{C})$ in Awake and Anesthesia

Data were expressed as mean \pm S.D.; Student's $t$-tests for comparison between anesthesia and awake for all patients. ${ }^{* *} p<0.01$ vs. awake; ns indicates no significance.

of volume expansion, or it was distributed in the interstitial space, causing postoperative tissue edema. ${ }^{2,3,18)}$ We found that the effect of surgery on Ringer's VE effect was similar to that of anesthesia, but there were no differences between the two kind of different surgeries.

During the experiment, the hemodynamics showed that inflammation hardly affected SBP, while for MAP and DBP, over the whole experiment, after infusion, during anesthesia or when awake, the patients in the Chronic group had higher indices than in those in Acute group. HR, in contrast, was higher in the Acute group than the Chronic group. The baseline hemodynamic characteristics were characterized by acute inflammation: increased heart rate and decreased peripheral resistance. In addition, in the study, the patients with acute inflammation were all patients with acute appendicitis undergoing emergency surgery, and most of the patients had elevated body temperature, accompanied by nausea and vomiting and other gastrointestinal symptoms, resulting in more severe dehydration than patients undergoing selective surgery. The results showed that, on the basis of hemodynamics, the hemodilution, VE, and VEE in the Acute group were all higher than in the Chronic. Despite the experience of anesthesia and infusion, the hemodynamic characteristics of the two groups were not changed. Specific reasons were perhaps as follows. In this study, fluid therapy was performed according to the body weight and not according to cardiac output (CO), stroke volume variation (SVV) or other indicators, called goaldirected fluid therapy (GDFT).

MAP and DBP in the acute group were significantly lower than those in the chronic group, HR was on the contrary, but almost all of the values were within the physiologically normal range. In this study, the early stage of inflammation in the acute group caused peripheral vasodilation, which resulted in a relatively insufficient volume compared with the chronic group, presenting the above hemodynamic characteristics; also, HR in the acute group was higher than that in the chronic group, which may also be related to high temperature. Even after fluid therapy, patients in the Acute group might still have had a lower blood volume relative to Chronic group. Neither anesthetics nor fluid infusion can combat low peripheral resistance in patients with acute inflammation, thus resulting in lower DBP and higher HR after infusion or during anesthesia (most anesthetics have vasodilation effects), compared to chronic.

This study had some limitations. First, the blood volume could have an impact on the distribution and clearance of infused fluid. ${ }^{2,19)}$ Vascular permeability might also promote fluid to distribute from the vascular space to the extravascular space. ${ }^{20,21)}$ In the present study, grouping was performed on the basis of SIRS diagnostic criteria and CRP values higher than normal. Thus, the patients with cholecystitis in the Chronic group were not in the acute phase of inflammation, showing no SIRS symptoms, and their CRP levels were within normal ranges, but the patients might still have had chronic inflammation and had frequent acute attacks. The VE of infused fluid might depend on the volume state or vascular permeability. For example, in sepsis, the infusion fluid was easily distributed to the interstitial space. ${ }^{7)}$ The specific mechanism remains unclear. In the present study, all patients in the acute group received antibiotics preoperatively, while all patients in the chronic group did not. Antibiotics reduce the degree of inflammation and may improve the permeability of blood vessels. After crystal infusion, there was no difference in the distribution of fluid outside blood vessels between the two groups, which may be related to the use of antibiotics in the acute group. In this study, under SIRS, the following points may be responsible for increased vascular permeability: Endothelial cell contraction or enhanced cytotoxicity; Direct injury to endothelial cells; Leukocyte mediated endothelial cell injury; High permeability of new capillary wall and local inflammatory cytokines increased so that the permeability of the vascular wall increased. We have had measured the serum level of inflammatory cytokines and we will report in the companion manuscript. A recent study ${ }^{15}$ showed that aquaporin 3 (AQP3) was involved in the regulation of pulmonary vascular permeability in sepsis, and antioxidants ${ }^{15,16)}$ can reduce pulmonary vascular permeability in sepsis and downregulate the expression of AQP3. Further studies are needed to confirm whether AQP3 expression is different in acute or 
Table 3. Comparison of Changes in Blood Pressure and HR between Groups

\begin{tabular}{|c|c|c|c|}
\hline & Chronic group & Acute group & $p$-Values \\
\hline \multicolumn{4}{|l|}{ MAP (mmHg) } \\
\hline Baseline during infusion $(0-35 \mathrm{~min})$ & $98 \pm 1488 \pm 17$ & $92 \pm 1684 \pm 18$ & 0.2920 .034 \\
\hline After infusion (40-120 min) & $90 \pm 16$ & $87 \pm 16$ & 0.010 \\
\hline During experiment $(0-120 \mathrm{~min})$ & $88 \pm 17$ & $84 \pm 17$ & 0.005 \\
\hline Awake & $89 \pm 17$ & $85 \pm 18$ & 0.034 \\
\hline Anesthesia & $86 \pm 17$ & $84 \pm 16$ & 0.076 \\
\hline \multicolumn{4}{|l|}{ SBP (mmHg) } \\
\hline Baseline & $139 \pm 26$ & $141 \pm 25$ & 0.843 \\
\hline During infusion $(0-35 \mathrm{~min})$ & $125 \pm 25$ & $124 \pm 25$ & 0.447 \\
\hline After infusion (4-120 min) & $121 \pm 23$ & $119 \pm 21$ & 0.488 \\
\hline During experiment $(0-120 \mathrm{~min})$ & $121 \pm 23$ & $120 \pm 22$ & 0.636 \\
\hline Awake & $123 \pm 25$ & $119 \pm 24$ & 0.156 \\
\hline Anesthesia & $119 \pm 22$ & $121 \pm 21$ & 0.430 \\
\hline \multicolumn{4}{|l|}{ DBP (mmHg) } \\
\hline Baseline & $75 \pm 10$ & $68 \pm 9$ & 0.031 \\
\hline During infusion $(0-35 \mathrm{~min})$ & $69 \pm 14$ & $66 \pm 13$ & 0.071 \\
\hline After infusion (40-120 min) & $71 \pm 13$ & $68 \pm 13$ & 0.010 \\
\hline During experiment $(0-120 \mathrm{~min})$ & $69 \pm 13$ & $66 \pm 13$ & 0.010 \\
\hline Awake & $69 \pm 13$ & $66 \pm 14$ & 0.028 \\
\hline Anesthesia & $69 \pm 14$ & $67 \pm 13$ & 0.137 \\
\hline \multicolumn{4}{|l|}{ HR (bpm) } \\
\hline Baseline & $82 \pm 10$ & $92 \pm 18$ & 0.044 \\
\hline During infusion (0-35 min) & $71 \pm 12$ & $80 \pm 17$ & $<0.001$ \\
\hline After infusion $(40-120 \mathrm{~min})$ & $66 \pm 11^{\# \#}$ & $75 \pm 16^{\# \#}$ & $<0.001$ \\
\hline During experiment $(0-120 \mathrm{~min})$ & $68 \pm 12$ & $78 \pm 17$ & $<0.001$ \\
\hline Awake & $70 \pm 12$ & $81 \pm 18$ & $<0.001$ \\
\hline Anesthesia & $66 \pm 11 * *$ & $76 \pm 15^{* *}$ & $<0.001$ \\
\hline
\end{tabular}

Data are expressed as the mean \pm S.D.; $* * p<0.01$ vs. awake; ${ }^{\# \#} p<0.01$ vs. during infusion $(0-35 \mathrm{~min})$.
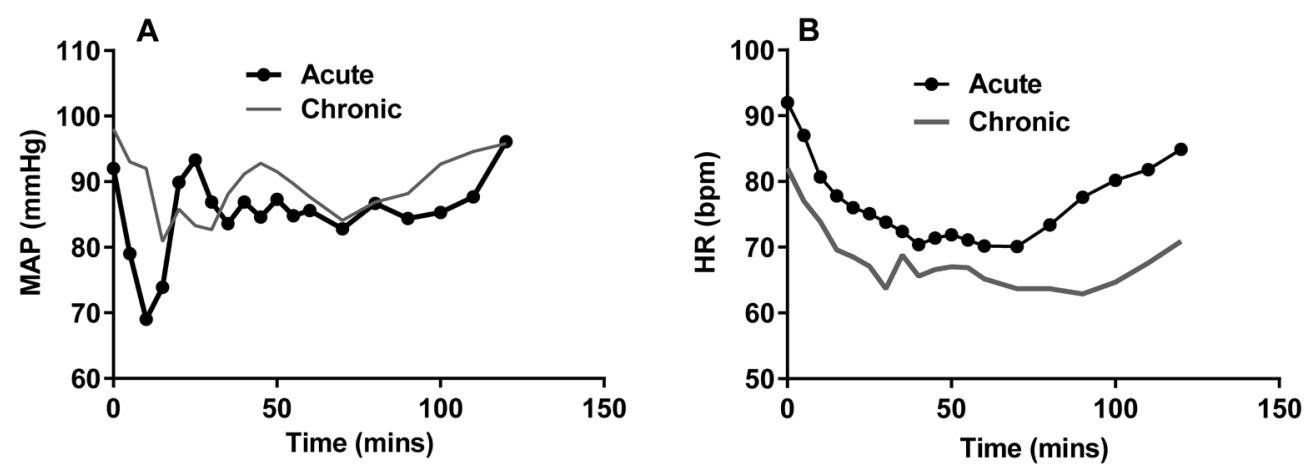

Fig. 4. The Trends for MAP (A) and HR (B) during and after Infusion of Ringer's Lactate Solution in Patients from the Chronic (Gray Line) and Acute (Black Line with Solid Circle) Groups

MAP, mean arterial blood pressure; HR, heart rate.

chronic inflammation. Second, the law of conservation of mass can be used to calculate the amount of intravascular or extravascular fluid, and the fluid expansion efficiency can also be directly calculated. ${ }^{3,4)}$ However, it cannot describe the dynamic equilibrium trend in fluid distribution between the intravascular and extravascular volume spaces. Volume kinetic analysis can reflect the specific distribution and clearance rate, but it cannot reflect VEE. ${ }^{14,22)}$ In this study, only one urine volume was collected at the end of experiment; thus, it was difficult to actually calculate the trend in fluid distribution between the intravascular and extravascular volume spaces. In addition, $\mathrm{CO}$ and SVV were not monitored in this study, so they could not reflect the individual patient's volume state.
Present studies showed that vasopressin/arginine vasopressin (ADH/AVP) plays an important role in renal water retention, mainly through arginine vasopressin AVP2 receptor (V2R) mediated AQP2 regulation. ${ }^{23)}$ Surgery and anesthesia can increase the reabsorption of water by the renal medullary collecting duct and reduce urine excretion through the AVP-V2RAQP2 axis. $^{24,25)}$

This present study only investigated the effects of acute or chronic inflammation on the distribution and clearance of infused crystalloids during anesthesia. There are some limitations in the study and the results may be suitable for guiding fluid therapy during surgical anesthesia for mild inflammation such as acute appendicitis or similar diseases. In this study, 
most of the patients with acute appendicitis surgery were operated on within $12 \mathrm{~h}$ of the onset of the disease, and no patients with chronic appendicitis were included. In further studies, the operation of chronic appendicitis and acute appendicitis patients will be compared, and the distribution and clearance situation after liquid treatment will be compared, so as to eliminate the influence of surgical factors. We just invested the effects of acute/chronic inflammation and anesthesia on the fluid shift of the infused fluid. We will explore the impact of surgery or surgical time on the fluid shift in the future studies.

In conclusion, this study showed that the plasma dilution, VE and VEE of patients with acute appendicitis (acute inflammation) after fluid infusion were higher than patients cholecystitis of cholethiasis (chronic inflammation). Especially under anesthesia or during surgery, VE increased; inflammation state, disease, or surgery performance had no effect on the extravascular distribution of infused fluid or its clearance through the kidneys. Hemodynamic characteristic showed that MAP decreased, and HR increased in patients with Acute inflammation, whether being awake or under anesthesia, during or after infusion. The results suggest that, for patients with acute inflammation, GDFT should be carried out according to the plasma volume state, and vasoconstrictor agents should be used properly to prevent fluid from accumulating in the interstitial space, leading to tissue edema after surgery and affecting the prognosis.

Acknowledgments This study was supported by Zhejiang Provincial Department of Science and Technology Fund (Grant No. LGF19H030011), Zhejiang Provincial Health Committee Fund (Grant Nos. 2018KY173, 2019306157), Shaoxing Science and Technology Bureau Fund (Grant No. 2017B70038), and Zhejiang Medical Association Foundation (2018zyc-A104), Shaoxing key discipine of anesthesiolgy (2019SZD04).

Author Contributions All authors listed in the manuscript have contributed to the plan of the study, analysis of the data, draft of the manuscript or critical revisions and have approved the final version of the manuscript.

Conflict of Interest The authors declare no conflict of interest.

\section{REFERENCES}

1) Moghadamyeghaneh Z, Phelan MJ, Carmichael JC, Mills SD, Pigazzi A, Nguyen NT, Stamos MJ. Preoperative dehydration increases risk of postoperative acute renal failure in colon and rectal surgery. J. Gastrointest. Surg., 18, 2178-2185 (2014).

2) Li YH, He R, Ying XJ, Hahn RG. Dehydration, haemodynamics and fluid volume optimization after induction of general anaesthesia. Clinics, 69, 809-816 (2014).

3) Li Y, Zhu S, Hahn RG. The kinetics of Ringer's solution in young and elderly patients during induction of general anesthesia with propofol and epidural anesthesia with ropivacaine. Acta Anaesthesiol. Scand., 51, 880-887 (2007).

4) Zhu GZ, Li YH, Ru GM, Ding Q. Comparison of the hemodynamics and dynamics of fluid shift of Ringer's solution before surgery in children and adults. Exp. Ther. Med., 13, 3146-3152 (2017).

5) Yue S, Xiaozhu H, Guomei R, Li Y. Effect of adrenergic alpha-1 receptor agonists on the volume kinetics of lactated Ringer's solu- tion in patients during laproscopic gynecological surgery. Chinese Journal of Critical Care Medicine, 8, 84-90 (2015).

6) Youjuan Q, Yuhong LI, Qiannan D, Guomei R. Effect of Esmolol on the distribution and clearance of lactated Ringer' s solution during general anesthesia. Zhejiang Medical Journal, 38, 1656-1661 (2016).

7) Li Y, Xiaozhu Z, Guomei R, Qiannan D, Hahn RG. The effects of vasoactive drugs on crystalloid fluid kinetics in septic sheep. PLOS $O N E$, 12, e0172361 (2017).

8) Beale RJ, Hollenberg SM, Vincent JL, Parrillo JE. Vasopressor and inotropic support in septic shock: an evidence-based review. Crit. Care Med., 32 (Suppl.), S455-S465 (2004).

9) Patel GP, Grahe JS, Sperry M, Singla S, Elpern E, Lateef O, Balk RA. Efficacy and safety of dopamine versus norepinephrine in the management of septic shock. Shock, 33, 375-380 (2010).

10) Ewaldsson CA, Vane LA, Kramer GC, Hahn RG. Adrenergic drugs alter both the fluid kinetics and the hemodynamic responses to volume expansion in sheep. J. Surg. Res., 131, 7-14 (2006).

11) Retzlaff JA, Tauxe WN, Kiely JM, Stroebel CF. Erythrocyte volume, plasma volume and lean body mass in adult men and women. Blood, 33, 649-661 (1969).

12) Zdolsek J, Li Y, Hahn RG. Detection of dehydration by using volume kinetics. Anesth. Analg., 115, 814-822 (2012).

13) Hahn RG, Li Y, Zdolsek J. Non-invasive monitoring of blood haemoglobin for analysis of fluid volume kinetics. Acta Anaesthesiol. Scand., 54, 1233-1240 (2010).

14) Li Y, Yi S, Zhu Y, Hahn RG. Volume kinetics of Ringer's lactate solution in acute inflammatory disease. Br. J. Anaesth., 121, 574580 (2018).

15) Drobin D, Hahn RG. Distribution and elimination of crystalloid fluid in pre-eclampsia. Clin. Sci., 106, 307-313 (2004).

16) Li YH, Lou XF, Bao FP. Dynamics of vascular volume and hemodilution of lactated Ringer's solution in patients during induction of general and epidural anesthesia. J. Zhejiang Univ. Sci. B, 7, 738-744 (2006).

17) Länne T, Lundvall J. Very rapid net transcapillary fluid absorption from skeletal muscle and skin in man during pronounced hypovolaemic circulatory stress. Acta Physiol. Scand., 136, 1-6 (1989).

18) Zhu G, Li Y, Ru G, Ding Q. Comparison of the hemodynamics and dynamics of fluid shift of Ringer's solution before surgery in children and adults. Exp. Ther. Med., 13, 3146-3152 (2017).

19) Li YH, Zhu HB, Zheng X, Chen HJ, Shao L, Hahn RG. Low doses of esmolol and phenylephrine act as diuretics during intravenous anesthesia. Crit. Care, 16, R18 (2012).

20) Ping W, Huiling W, Yu Z, Li T, Liu L. Role of aquaporin 3 in pulmonary vascular leakage after sepsis and effect of SS-31 in the process in vitro and in vivo. Journal of Third Military Medical University, 39, 111-115 (2017).

21) Pellavio G, Rui M, Caliogna L, Martino E, Gastaldi G, Collina S, Laforenza U. Regulation of aquaporin functional properties mediated by the antioxidant effects of natural compounds. Int. J. Mol. Sci., 18, 2665 (2017).

22) Hahn RG, Drobin D, Zdolsek J. Distribution of crystalloid fluid changes with the rate of infusion: a population-based study. Acta Anaesthesiol. Scand., 60, 569-578 (2016).

23) Cowen LE, Hodak SP, Verbalis JG. Age-associated abnormalities of water homeostasis. Endocrinol. Metab. Clin. North Am., 42, 349-370 (2013).

24) Juul KV. The evolutionary origin of the vasopressin/V2-type receptor/aquaporin axis and the urine-concentrating mechanism. Endocrine, 42, 63-68 (2012).

25) Ogura T, Morita K, Takeuchi M, Otsuka F, Iwasaki T, Oe K, Matsuura K, Tobe K, Mimura Y, Kishida M, Makino H, Hirakawa M. Relationship between adrenomedullin and vasopressin-aquaporin system under general anesthesia. Horm. Res., 59, 30-34 (2003). 